\title{
Los Objetivos de Desarrollo Sostenible dentro del deporte social latinoamericano. Una organización-metodología desde México
}

\author{
The Sustainable Development Goals within Latin American \\ social sport. An organization-methodology from Mexico
}

\section{Daniel Añorve Añorve* \\ y José Guadalupe Flores Palafox**}

\footnotetext{
Profesor e investigador del Departamento de Estudios Políticos y de Gobierno de la Universidad de Guanajuato. Miembro del Sistema Nacional de Investigadores (nivel 2). Doctor en Relaciones Internacionales por la Universidad Nacional Autónoma de México (UNAM). Maestría en Ciencia Política en York University, Canadá.

-danorve@gmail.com

http://orcid.org/0000-0002-

9603-9676

** Estudiante de la Licenciatura en Ciencia Política, Universidad de Guanajuato. Becario del proyecto Tiro en Braille.

·jg.florespalafox@ugto.mx http://orcid.org/0000-00019195-0877
}

RECIBIDO: 4.8 .2021

\section{Resumen}

El deporte social, globalmente conocido como Deporte para el Desarrollo y la Paz (SDP) y la Agenda 2030/los Objetivos de Desarrollo Sostenible (ODS) son dos acontecimientos globales que marcan el inicio del siglo XXI. A diferencia de los Objetivos de Desarrollo del Milenio, la Agenda 2030 contempla al deporte como unas de las actividades culturales que pueden coadyuvar a la consecución de los ODS. En América Latina y el Caribe existe un creciente interés en el deporte social como un instrumento para lograr tanto la pacificación como la inclusión social. Basándose en una revisión minuciosa de la plataforma líder global Sportanddev.org, se identifican 79 organizaciones ad hoc en América Latina y el Caribe, solo diez de las cuales contemplan su vinculación con los ODS. Se analiza una organización mexicana pionera - Tiro en Braille- como la única organización SDP regional que, además de contemplar entre sus objetivos los ODS, trabaja en múltiples aspectos de inclusión: género, discapacidad, poblaciones universitarias, creación de deportes según un esquema de plus sport y recuperación de juegos y deportes tradicionales o autóctonos. Se explica la forma en que la metodología desarrollada por Tiro en Braille es susceptible de replicarse en diversos países de la región. 
Palabras clave: deporte, integración social, América Latina, Caribe.

\section{Abstract}

Social sport, globally known as Sport for Development and Peace (SDP) and the 2030 Agenda and its Sustainable Development Goals (SDG) are two global events that mark the beginning of the 21st Century. In contrast with the Millennium Development Goals, the 2030 Agenda considers sport as one of the cultural activities that can contribute to the achievement of the SDG. In Latin America and the Caribbean there is a growing interest in social sport as a tool to achieve both pacification of countries and social inclusion. Based on a detailed analysis, it is possible to identify seventy-nine ad hoc organizations working in Latin America and the Caribbean. Only ten connect to the SDG. This paper analyzes Tiro en Braille (Braille Shot), a pioneer Mexican SDP organization, as the only regional organization that besides working towards the SDG, includes several facets of social inclusion: gender, disability, university populations, the purposely design of new sports under a plus sport approach, as well as the recovery of traditional/indigenous games/sports. It is explained how Tiro en Braille's methodology is susceptible to be replied in different countries of the region.

Keywords: sport, social integration, Latin America, Caribbean.

\section{Introducción}

Tanto el deporte para el desarrollo y la paz (conocido como SDP por sus siglas en inglés) como la Agenda 2030 y los 17 Objetivos de Desarrollo Sostenible (ODS) son dos acontecimientos globales que marcan las dos primeras décadas del siglo XXI. A diferencia de los Objetivos de Desarrollo del Milenio (ODM), la Agenda 2030 contempla al deporte como unas de las actividades culturales que pueden coadyuvar a la consecución de la Agenda 2030 en su conjunto y de algunos de los ODS. En América Latina y el Caribe existe un creciente interés académico, gubernamental, social y empresarial en el llamado deporte social, ${ }^{1}$ ya sea para lograr la tan anhelada pacificación social, ${ }^{2}$ para

1 Aunque resulta evidente que todo deporte es social (tal como sucede con cualquier actividad de creación humana), se suele hablar de deporte social en contraposición al deporte de elite, el deporte profesional y el deporte de alto rendimiento, que presentan las siete características del deporte moderno mencionadas por Allen Guttmann en su obra clásica de 1978.

2 En México, la actual administración federal encabezada por Andrés Manuel López Obrador ha decidido cambiar de paradigma para pacificar al país. La idea es adoptar un enfoque menos punitivo que el puesto en marcha por los gobiernos que lo precedieron, referidos comúnmente como neoliberales. La apuesta 
promover la inclusión social, o bien, desde el campo empresarial, como parte del portafolio de la llamada Responsabilidad Social Empresarial (RSE) o corporativa. ${ }^{3}$

Es una tarea prácticamente imposible identificar todas y cada una de las iniciativas SDP existentes en la región. Se considera que un buen punto de partida es la plataforma global líder Sportanddev.org, domiciliada en Suiza operada por la Academia Suiza para el Desarrollo y con participación de diversas agencias para el desarrollo, tanto deportivo como general: el Alto Comisionado de las Naciones Unidas para los refugiados (UNHCR), el Comité Olímpico Noruego y la UEFA.

Dos preguntas orientan la investigación: 1) ¿Qué características (y deficiencias) tienen las organizaciones SDP latinoamericanas y del Caribe que trabajan vinculadas a los ODS? 2) ¿Qué aspectos de Tiro en Braille la convierten en una organización SDP susceptible de trascender las fronteras mexicanas?

El trabajo se divide en cinco apartados. El primero hace una breve introducción al SDP, a la literatura SDP en América Latina y a la vinculación entre el SDP y los ODS. El segundo introduce al lector en la plataforma global Sportanddev.org, además de explicar el procesamiento de la información. Un tercer apartado examina las organizaciones SDP que operan en la región de América Latina y el Caribe, concentrándose en el análisis minucioso de aquellas diez que contemplan la vinculación con la Agenda 2030/ODS. En el cuarto apartado se presenta el estudio de caso seleccionado: Tiro en Braille, una de las siete organizaciones SDP mexicanas, que destaca en el país y en la región porque no se limita a atender la Agenda 2030/ODS, sino que además contempla entre sus objetivos los ODS y el trabajo en múltiples aspectos de inclusión social: género, discapacidad, poblaciones universitarias, creación de deportes bajo un esquema de plus sport y recuperación de juegos y deportes tradicionales y autóctonos. En el quinto apartado se explica la principal aportación de la organización mexicana Tiro en Braille: su metodología, susceptible de replicarse en diversos países de la región a un bajo costo y facilitando la apropiación innovadora y autónoma de quienes la repliquen.

\section{El SDP y la literatura especializada en América Latina y el Caribe}

La literatura en torno a la sociología del deporte está ya consolidada en las ciencias sociales, al menos en el Norte global. Existe una vasta literatura en torno al SDP (Coalter,

de la actual administración reside en abrir todo tipo de espacios de convivencia constructiva para la juventud, entre ellos el deporte con un enfoque de política social.

3 Desde luego, los intereses corporativos suelen estar ligados a la expansión de sus mercados, a la atracción de fuerza de trabajo, a la mejora de la imagen corporativa de las empresas que hacen donaciones o encabezan programas y, por supuesto, a la reducción o condonación de impuestos. 
2007, 2010; Levermore, 2011; Hartmann y Kwauk, 2011; Darnell y Hayhurst, 2011; Mwaanga y Mwansa, 2014; Hatton, 2015; Burnett, 2015; Darnell et al., 2018; Giulianotti et al., 2019, solo por mencionar algunos trabajos), así como revistas ad hoc dentro de los índices más prestigiosos; por ejemplo, Sport Education and Society, International Review for the Sociology of Sport, Journal of Sport and Social Issues, Sociology of Sport Journal, Sport in Society, Journal of the Philosophy of Sport, International Journal of the History of Sport, etcétera. En dichas revistas, el sport for development and peace resulta un término clave común.

¿Qué se entiende por SDP? Un punto de inicio útil es la taxonomía formulada por Coalter (2007), que diferencia los tres grandes enfoques para entender el deporte: 1) las formas tradicionales del deporte; 2) el enfoque sport plus, en el cual los deportes son adaptados y frecuentemente acrecentados con el apoyo de programas paralelos, para así maximizar el potencial de objetivos de desarrollo contemplados por Naciones Unidas (en su momento los ODM o actualmente los ODS); el énfasis está en que el deporte crezca y se fortalezca con el apoyo de los programas paralelos; 3) el enfoque plus sport, que usa la popularidad del deporte como anzuelo para atraer a la gente a programas de educación y capacitación. El énfasis rara vez se halla en el deporte per se; más bien, el deporte es un vehículo para la consecución del desarrollo no deportivo.

La aproximación dominante, como una institución social moderna (Guttmann, 1978), suele tener carácter excluyente y ofrece limitadas posibilidades para lograr la inclusión. Actualmente, dentro del SDP, la opción sport plus parece ser la predilecta de los intereses corporativos, como parte de sus estrategias de responsabilidad social empresarial (RSE) (Paramaio-Salcines et al., 2013). Mientras tanto, la aproximación plus sport es similar a lo que se conoce como sport-for-all: además de desafiar al deporte moderno, pugna por su deconstrucción. Hatton (2015, p. 27) argumenta que el SDP no hace referencia a un tipo de organización ni tampoco a un sector en sí. Giulianotti et al. (2019) señalan que, desde los primeros años del siglo XXI, el SDP se ha convertido en un campo fuertemente institucionalizado, con sus propios distintivos, y que de forma creciente se conecta con las relaciones internacionales y con el sector del desarrollo global; es respaldado por organizaciones internacionales como la ONU y la Commonwealth. Como movimiento, el SDP es un producto del nuevo milenio. La ONU (United Nations, 2003) adoptó en noviembre de 2003, en el seno de la Asamblea General, una resolución que afirma su compromiso con el deporte como medio para promover la educación, la salud, el desarrollo y la paz, y para incluir el deporte y la educación física como herramientas que contribuyan al logro de los Objetivos de Desarrollo del Milenio (ODM). Para Burnett (2015, p. 385) el deporte para el desarrollo «nació» con la declaración en 2005 del Año Internacional del Deporte y la Educación Física (Melo, 2018).

Respecto al vínculo entre el SDP y los ODS, la Asamblea General de las Naciones Unidas adoptó la resolución 70/1, Transforming Our World: the 2030 Agenda for Sustainable Development, en noviembre de 2015 (United Nations General Assembly [UNGA], 
2015, p. 2). Aunque el deporte no se incluyó explícitamente en ninguno de los 17 ODS, la declaración inicial contiene un extracto que lo menciona:

El deporte es un importante facilitador del desarrollo sostenible. Reconocemos la contribución del deporte para la consecución del desarrollo y la paz en lo que toca a la promoción de la tolerancia y el respeto, además de las contribuciones que hace al empoderamiento de las mujeres y de la juventud, los individuos y las comunidades, además de a la salud, la educación y los objetivos de inclusión social.

Lindsey y Darby (2019) destacan que los ODS marcan la primera vez que una política de desarrollo global, por su amplitud, incluyó al deporte, y advierten una escasez de trabajos académicos que analicen sustancialmente la relación entre el deporte y los ODS. Consideran que hay ODS con metas específicas que tocan problemas claramente asociados con el deporte: la discriminación contra las mujeres (meta 5.1), el abuso y la violencia contra los niños (meta 16.2) y la corrupción y los sobornos (meta 16.5). También observan que los ODS contrastan con los ODM, toda vez que los primeros se definen como «integrados e indivisibles». En contraposición, los ODM estaban enfocados en resultados independientes y las metas eran relativamente aisladas del cumplimiento de otras.

Nunes et al. (2016) reconocen la importancia de diversos ODS para la consecución de metas específicas del ODS 3 (salud). Lindsey y Darby (2019) otorgan un lugar central al ODS 4 (educación) y consideran que la meta 4.7 tiene una gran relevancia más allá de la educación formal, al promover una amplia concepción de la educación para el desarrollo sostenible, que incluye elementos tales como estilos de vida sostenible, ciudadanía, equidad de género, paz y derechos humanos (UNGA, 2015, p. 17). Se trata, en síntesis, de «habilidades para la vida» que los proyectos SDP han buscado desarrollar.

En cuanto a la implementación práctica de los ODS dentro del SDP, representantes del futbol nórdico se reunieron en junio de 2017 con el primer ministro noruego para anunciar su apoyo a los ODS (Sundelin et al., 2017). Se asegura que es preciso ir más allá de las agencias de la ONU y del movimiento olímpico, por lo cual se requiere formar sociedades con órganos de gobernanza, la industria corporativa, las ONG y la Academia (Metropole Lémanique, 2017). Con la adopción de la Agenda 2030, se transita desde sectores específicos hacia una aproximación intersectorial y holística, y la agenda global toma en cuenta a socios extradeportivos (Metropole Lémanique, 2017).

El movimiento SDP no está libre de críticas. En términos generales, Añorve (2021) identifica cinco falencias dentro del mundo del SDP: 1) la mayoría de las iniciativas están relacionadas con el futbol; 2) se advierte una estrechez geográfica, tanto en términos académicos como en la práctica de campo; 3) persiste una división norte/sur; 4) es marcado el énfasis en la atención de poblaciones altamente marginadas, y 5) hay escasa 
conexión entre el movimiento SDP y las legislaciones nacionales, además de que el vínculo con la Agenda 2030 no deja de ser precario, muchas veces simplemente discursivo.

\section{La bibliografía en América Latina y en México}

En comparación con lo ocurrido con la sociología del deporte en otras latitudes —el mundo anglosajón y Europa occidental—-, el desarrollo de esta subdisciplina en América Latina ha sido reciente, lento, menos profundo y de menor visibilidad. A pesar de la inclusión del deporte en la agenda política de los países latinoamericanos, no fue hasta 2010 que se consolidó un espacio para la discusión seria (Asociación Latinoamericana de Estudios Socioculturales del Deporte [ALESDE], 2010). En paralelo a su fundación y consolidación, ALESDE decidió lanzar su revista oficial en 2011 (Marchi, 2015). La Asociación y su revista oficial Journal of the Latin American Socio-cultural Studies of Sport (JLASSS) representan un vehículo regional para reducir las brechas con organizaciones (como la International Sports Sciences Association [ISSA]) y académicamente con la International Review for the Sociology of Sport y el Sociology of Sport Journal, líderes en la subdisciplina. Un análisis propio, en julio de 2021, permite ver que lo que se conoce genéricamente en inglés como SDP o deporte social ha estado ausente en el JLASSS.

Al buscar la palabra desarrollo, sin adjetivación, dentro del título de los artículos, se arrojan dos resultados, ambos sin conexión alguna con lo que es el SDP. La búsqueda en los resúmenes arroja cuatro resultados, uno de ellos en clara referencia al desarrollo sostenible (Inglés y Puig, 2013). Al buscar en las palabras clave solo aparece el mismo trabajo de Inglés y Puig (2013). De hecho, el lugar marginal asignado al SDP es fácilmente contrastable con lo que ocurre con ISSA, pues mientras el SDP es uno de los ejes temáticos de ISSA, ALESDE no lo incluye dentro de sus 25 ejes temáticos. Entre los números temáticos del JLASSS, ninguno aborda el deporte para el desarrollo y la paz; no obstante, el número 1, de 2019, es dedicado al deporte social, con tres trabajos especializados (Barboza y Marinho, 2019; Kravchychyn et al., 2019; Toledo y Silva, 2019). Tres de 139 artículos publicados en la primera década de vida del JLASSS representan el 2,16\%, lo que confirma la marginalidad de los estudios que abordan el deporte social o el SDP.

Por lo que toca a México, al revisarse las revistas sobre deportes dentro del área de ciencias sociales en Latindex, para Iberoamérica, aparecen 232 revistas (búsqueda del 23 de abril de 2020), argentinas, españolas, portuguesas, cubanas, venezolanas, brasileñas, uruguayas, chilenas, costarricenses, ecuatorianas, colombianas y nicaragüenses. México registra tan solo una revista -Deporte, Ciencia \& Técnica-, que se publicó brevemente, de 1998 a 1999. En Scielo, la búsqueda combinada de las palabras deporte y desarrollo arroja diez resultados para México, ninguno de los cuales corresponde al 
SDP. No hay nada que trate sobre los Objetivos de Desarrollo Sostenible ni las agendas globales, así como tampoco figuran trabajos que versen sobre el SDP explícitamente.

\section{Revisión de la plataforma Sportanddev.org}

Dentro de los recursos en materia de SDP, quizá ninguno tenga la centralidad de Sportanddev.org. Para el 10 de abril de 2020, 1017 organizaciones aparecían en el listado de la plataforma. Esto contrasta con los 448 registros existentes en 2013 (Mwaanga y Mwansa, 2014). No obstante el crecimiento en términos absolutos, la exclusión es visible desde otra óptica. De las 1017 organizaciones listadas, 517 están basadas en Europa, América del Norte (excluido México), Australia, Nueva Zelanda y África. Decenas de países brillan por su ausencia. Hablar del mundo SDP es hablar de una geopolítica del mundo del Sportland. Al revisar el listado se aprecia la concentración, completamente desproporcional, que tienen África y Europa dentro de dicha plataforma.

Este artículo parte de un análisis regional y posteriormente nacional (para México) de las organizaciones en la plataforma Sportanddev.org desde diversos desgloses: geográfico, de sectores o grupos vulnerables o minoritarios que atienden (incluyen) las organizaciones SDP que operan en la región. Toda vez que el énfasis del artículo se centra en la Agenda 2030/ODS, el análisis propiamente cualitativo se hará para aquellas organizaciones de América Latina y el Caribe, y particularmente de México, que incluyen entre sus iniciativas la Agenda 2030/ODS, diferenciando entre aquellas organizaciones que se limitan a mencionarla (de forma enunciativa) y aquellas que la aplican dentro de sus iniciativas (de forma sustantiva).

\section{Análisis regional (América Latina y el Caribe) y nacional (México) de las organizaciones SDP dentro de Sportanddev.org}

Un análisis regional muestra que América Latina y el Caribe cuenta con 79 organizaciones domiciliadas en la región, lo cual representa un 7,76\% del total mundial. La tabla 1 permite conocer el desglose por país dentro de la región. México cuenta con 7 de las 79 organizaciones, o sea, un 8,86\% de las organizaciones de la región o el 0,68\% del total mundial. 
Tabla 1. Organizaciones SDP domiciliadas en América Latina y el Caribe (Sportanddev.org)

\begin{tabular}{|c|c|c|}
\hline País & $\begin{array}{l}\text { Número de } \\
\text { organizaciones }\end{array}$ & $\begin{array}{l}\text { Número y nombre de las organizaciones } \\
\text { que trabajan con los ODS }\end{array}$ \\
\hline Brasil & 10 & $\begin{array}{l}2 \text { (Associação Jadir de Taekwondo y Capoeira } \\
\text { Congo) }\end{array}$ \\
\hline Colombia & 17 & $\begin{array}{l}3 \text { (Colombianitos, Fórmula Sonrisas y Futbol con } \\
\text { Corazón) }\end{array}$ \\
\hline Trinidad y Tobago & 3 & $\begin{array}{l}1 \text { (Caribbean Sport and Development Agency } \\
\text { [CSDA]) }\end{array}$ \\
\hline Chile & 3 & 1 (Ruby para Todos Chile) \\
\hline Bolivia & 2 & \\
\hline México & 7 & 2 (Fundación Televisa y Tiro en Braille) \\
\hline Venezuela & 2 & \\
\hline Argentina & 4 & \\
\hline Nicaragua & 2 & \\
\hline Honduras & 3 & \\
\hline Dominica & 1 & \\
\hline Haití & 7 & 1 (Hav Serve in Haiti) \\
\hline Costa Rica & 2 & \\
\hline El Salvador & 1 & \\
\hline Ecuador & 2 & \\
\hline Perú & 4 & \\
\hline Puerto Rico & 1 & \\
\hline Guyana & 1 & \\
\hline Uruguay & 1 & \\
\hline Panamá & 1 & \\
\hline Santa Lucía & 1 & \\
\hline Paraguay & 1 & \\
\hline Surinam & 1 & \\
\hline Barbados & 1 & \\
\hline Jamaica & 1 & \\
\hline Total & 79 & 10 \\
\hline
\end{tabular}


Tabla 2. Las organizaciones SDP mexicanas dentro de Sportanddev.org

\begin{tabular}{|c|c|c|c|c|c|c|c|c|c|c|c|}
\hline Organización & $\begin{array}{l}\text { Agenda } \\
2030 / 0 D S\end{array}$ & Fútbol & $\begin{array}{l}\text { Otro } \\
\text { deporte }\end{array}$ & $\begin{array}{l}\text { Creación } \\
\text { de otro } \\
\text { deporte }\end{array}$ & Género & Discapacidad & $\begin{array}{l}\text { Juegos/ } \\
\text { deporte } \\
\text { tradicional }\end{array}$ & $\begin{array}{l}\text { Estudian- } \\
\text { tes uni- } \\
\text { versitarios }\end{array}$ & $\begin{array}{l}\text { Tipo de } \\
\text { patrocinador }\end{array}$ & Entidad & Observaciones \\
\hline $\begin{array}{l}\text { Promotora } \\
\text { Mexicana de } \\
\text { Valores } \\
\text { Deportivos } \\
\text { A. C. }\end{array}$ & $x$ & $\checkmark$ & $\checkmark$ & $x$ & $\checkmark$ & $x$ & $\checkmark$ & $x$ & $\begin{array}{l}\text { A. C., donativos } \\
\text { individuales y } \\
\text { corporativos }\end{array}$ & Tamaulipas & $\begin{array}{l}\text { Tiene una tienda de } \\
\text { artículos deportivos. } \\
\text { No explica cómo atiende } \\
\text { las problemáticas sociales } \\
\text { enunciadas }\end{array}$ \\
\hline $\begin{array}{l}\text { Capoeira para } \\
\text { Todos A. C. }\end{array}$ & $x$ & $x$ & $\checkmark$ & $x$ & $\checkmark$ & N/D & $\checkmark$ & $\checkmark$ & $\begin{array}{l}\text { Acepta donativos } \\
\text { individuales }\end{array}$ & $\begin{array}{l}\text { Aguas } \\
\text { Calientes }\end{array}$ & $\begin{array}{l}\text { Se habla de } \\
\text { empoderamiento y combate } \\
\text { a la violencia (sin detalles) }\end{array}$ \\
\hline $\begin{array}{l}\text { United } \\
\text { Noses.com }\end{array}$ & $x$ & $N / D$ & $\mathrm{~N} / \mathrm{D}$ & $x$ & $\mathrm{~N} / \mathrm{D}$ & N/D & $x$ & N/D & $\begin{array}{l}\text { Movimiento } \\
\text { internacional. } \\
\text { No queda claro } \\
\text { el patrocinio }\end{array}$ & Guadalajara & $\begin{array}{l}\text { El componente deportivo no } \\
\text { queda claro; se diluye entre } \\
\text { generalidades acerca de la } \\
\text { felicidad }\end{array}$ \\
\hline $\begin{array}{l}\text { Tiro en } \\
\text { Braille }\end{array}$ & $\checkmark$ & $x$ & $x$ & $\checkmark$ & $\checkmark$ & $\checkmark$ & $\checkmark$ & $\checkmark$ & $\begin{array}{l}\text { Universidad de } \\
\text { Guanajuato, } \\
\text { pequeñas y } \\
\text { medianas } \\
\text { empresas locales }\end{array}$ & o. M. & $\begin{array}{l}\text { Véanse detalles en la cuarta } \\
\text { sección }\end{array}$ \\
\hline $\begin{array}{l}\text { Fundación } \\
\text { Televisa }\end{array}$ & $\checkmark$ & $\checkmark$ & $x$ & $x$ & $\checkmark$ & $\checkmark$ & $x$ & $x$ & $\begin{array}{l}\text { Apoyos } \\
\text { corporativos }\end{array}$ & $\begin{array}{l}\text { Ciudad de } \\
\text { México }\end{array}$ & $\begin{array}{l}\text { La iniciativa insignia es Gol } \\
\text { por México }\end{array}$ \\
\hline Blacksqrl & $x$ & $\checkmark$ & $x$ & $x$ & $x$ & $\checkmark$ & $x$ & $x$ & Programa de RSE & $\begin{array}{l}\text { Ciudad de } \\
\text { México }\end{array}$ & $\begin{array}{l}\text { Compañía de logística } \\
\text { formada por veteranos } \\
\text { de guerra. El único } \\
\text { documento deportivo habla } \\
\text { de trabajo con la } \\
\text { Confederación } \Lambda \text { siática } \\
\text { de Fútbol. No tiene } \\
\text { iniciativas en México }\end{array}$ \\
\hline $\begin{array}{l}\text { Street Soccer } \\
\text { Mexico A. C }\end{array}$ & $x$ & $\checkmark$ & $x$ & $x$ & $\checkmark$ & $x$ & $x$ & $x$ & $\begin{array}{l}\text { Sí, además } \\
\text { alianzas } \\
\text { gubernamentales }\end{array}$ & $\operatorname{CDMX}$ & $\begin{array}{l}\text { Inspirado en el Homeless } \\
\text { World Cup. Funciona en } \\
\text { cuatro estados de México }\end{array}$ \\
\hline
\end{tabular}


Tabla 3. Las organizaciones SDP en América Latina y el Caribe que trabajan con la Agenda 2030/ODS (Sportanddev.org)

\begin{tabular}{|c|c|c|c|c|c|c|c|c|c|c|c|}
\hline Organización & $\begin{array}{l}\text { Agenda } \\
\text { 2030/ODS } \\
\text { (enunciativo } \\
\text { o sustantivo) }\end{array}$ & Futbol & $\begin{array}{l}\text { Otro } \\
\text { deporte }\end{array}$ & $\begin{array}{l}\text { Creación de } \\
\text { otro deporte }\end{array}$ & Género & Discapacidad & \begin{tabular}{|l} 
Juegos o \\
deportes \\
tradicionales
\end{tabular} & $\begin{array}{l}\text { Estudiantes } \\
\text { universitarios }\end{array}$ & $\begin{array}{l}\text { Tipo de } \\
\text { patrocinador }\end{array}$ & País & Observaciones \\
\hline $\begin{array}{l}\text { Associação } \\
\text { Jadir de } \\
\text { Taekwondo }\end{array}$ & Sustantivo & $\mathrm{x}$ & $\checkmark$ & $\mathrm{x}$ & $\checkmark$ & $\mathrm{X}$ & $\mathrm{X}$ & $\mathrm{X}$ & $\begin{array}{l}\text { Apoyos públicos y } \\
\text { privados }\end{array}$ & Brasil & Dos décadas de existencia \\
\hline $\begin{array}{l}\text { Capoeira } \\
\text { Congo }\end{array}$ & Sustantivo & $\mathrm{x}$ & $\checkmark$ & $\mathrm{X}$ & $\checkmark$ & $\mathrm{X}$ & $\checkmark$ & $\checkmark$ & $\begin{array}{l}\text { Predominio de apoyos } \\
\text { públicos, no solo } \\
\text { brasileños. Un } \\
\text { patrocinador } \\
\text { corporativo, Canon }\end{array}$ & $\begin{array}{l}\text { Brasil } \\
\text { (opera en } \\
\text { países } \\
\text { africanos) }\end{array}$ & $\begin{array}{l}\text { La capoeira es patrimonio } \\
\text { intangible (UNESCO). } \\
\text { Participan Brasil, Canadá, } \\
\text { UNICEF y AMADE-Mondiale }\end{array}$ \\
\hline Colombianitos & Sustantivo & $\checkmark$ & $\checkmark$ & $\mathrm{X}$ & $\checkmark$ & $\mathrm{x}$ & $\mathrm{X}$ & $\mathrm{X}$ & $\begin{array}{l}\text { Fuerte apoyo } \\
\text { corporativo } \\
\text { (transnacional), } \\
\text { aunque también } \\
\text { público. }\end{array}$ & Colombia & $\begin{array}{l}\text { Creada en } 2001 \text {. Su } \\
\text { metodología, "Juguemos por } \\
\text { la paz", ha despertado interés } \\
\text { de Direct TV, ESPN y FIFA; } \\
\text { goza del reconocimiento del } \\
\text { BID y la Unesco }\end{array}$ \\
\hline $\begin{array}{l}\text { Fórmula } \\
\text { Sonrisas }\end{array}$ & $\begin{array}{l}\text { Enunciativo } \\
\text { (ODM) }\end{array}$ & $\checkmark$ & $\checkmark$ & $\mathrm{X}$ & $\checkmark$ & $\mathrm{X}$ & $\mathrm{X}$ & $\mathrm{x}$ & $\begin{array}{l}\text { Apoyos corporativos } \\
\text { (sector automotor y } \\
\text { deportivo), aunque } \\
\text { también públicos }\end{array}$ & Colombia & $\begin{array}{l}\text { Fundada por el piloto Juan } \\
\text { Pablo Montoya en } 2003\end{array}$ \\
\hline $\begin{array}{l}\text { Futbol con } \\
\text { Corazón }\end{array}$ & Sustantivo & $\checkmark$ & $\mathrm{X}$ & $\mathrm{X}$ & $\checkmark$ & $\mathrm{X}$ & $\mathrm{X}$ & $\mathrm{X}$ & $\begin{array}{l}\text { Apoyos corporativos } \\
\text { (transnacionales), } \\
\text { públicos. Gran énfasis } \\
\text { en la «fuerza del } \\
\text { mercado» }\end{array}$ & $\begin{array}{l}\text { Colombia } \\
\text { (opera en } \\
\text { Costa Rica, } \\
\text { Colombia, } \\
\text { Ecuador, } \\
\text { Panamá y } \\
\text { Estados } \\
\text { Unidos) }\end{array}$ & $\begin{array}{l}\text { Metodología «Futbol para la } \\
\text { paz» (futbol mixto, sin } \\
\text { mismo partido; no hay } \\
\text { suplentes). Metodología } \\
\text { reflexiva. Cuenta con una app. } \\
\text { Opera desde 2007. Oferta } \\
\text { cursos y diplomados. Miden } \\
\text { impactos }\end{array}$ \\
\hline $\begin{array}{l}\text { Caribbean } \\
\text { Sport and } \\
\text { Development } \\
\text { Agency } \\
\text { (CSDA) }\end{array}$ & Enunciativo & $\checkmark$ & $\checkmark$ & $\begin{array}{l}X \text { (aunque } \\
\text { plantea } \\
\text { adaptaciones } \\
\text { para } \\
\text { discapacitados) }\end{array}$ & $\checkmark$ & $\checkmark$ & $\mathrm{X}$ & \begin{tabular}{|l|}
$X$ (aunque \\
contempla \\
posibles \\
alianzas con \\
universitarios)
\end{tabular} & $\begin{array}{l}\text { ONG con múltiples } \\
\text { alianzas } \\
\text { internacionales, sobre } \\
\text { todo dentro de la } \\
\text { Commonwealth }\end{array}$ & $\begin{array}{l}\text { Trinidad y } \\
\text { Tobago, } \\
\text { pero opera } \\
\text { en } 13 \\
\text { territorios } \\
\text { caribeños }\end{array}$ & $\begin{array}{l}\text { Fuerte énfasis en la salud } \\
\text { preventiva y el covid-19. } \\
\text { Pone al deporte competitivo a } \\
\text { la par del deporte social }\end{array}$ \\
\hline $\begin{array}{l}\text { Fundación } \\
\text { Televisa }\end{array}$ & Sustantivo & $\checkmark$ & $\mathrm{x}$ & $\mathrm{X}$ & $\checkmark$ & $\mathrm{X}$ & $\mathrm{X}$ & $\mathrm{X}$ & $\begin{array}{l}\text { Sí, trabaja con GNP } \\
\text { (sector de seguros) }\end{array}$ & México & $\begin{array}{l}\text { Gol por México funciona a } \\
\text { través de las ligas } \\
\text { profesionales (varonil y } \\
\text { femenina) }\end{array}$ \\
\hline
\end{tabular}


(continuación)

\begin{tabular}{|c|c|c|c|c|c|c|c|c|c|c|c|}
\hline Organización & $\begin{array}{l}\text { Agenda } \\
\text { 2030/ODS } \\
\text { (enunciativo } \\
\text { o sustantivo) }\end{array}$ & Futbol & $\begin{array}{l}\text { Otro } \\
\text { deporte }\end{array}$ & $\begin{array}{l}\text { Creación de } \\
\text { otro deporte }\end{array}$ & Género & Discapacidad & $\begin{array}{l}\text { Juegos o } \\
\text { deportes } \\
\text { tradicionales }\end{array}$ & $\begin{array}{l}\text { Estudiantes } \\
\text { universitarios }\end{array}$ & $\begin{array}{l}\text { Tipo de } \\
\text { patrocinador }\end{array}$ & País & Observaciones \\
\hline Tiro en Braille & Sustantivo & $\mathrm{x}$ & $\checkmark$ & $\checkmark$ & $\checkmark$ & $\checkmark$ & $\checkmark$ & $\checkmark$ & $\begin{array}{l}\text { No. Plantea alianzas } \\
\text { con pymes locales y } \\
\text { con sector público }\end{array}$ & México & $\begin{array}{l}\text { Iniciativa de la Universidad } \\
\text { de Guanajuato }\end{array}$ \\
\hline $\begin{array}{l}\text { HavServe in } \\
\text { Haiti }\end{array}$ & $\begin{array}{l}\text { Enunciativo. } \\
\text { Pese al fuerte } \\
\text { compromiso } \\
\text { de la } \\
\text { organiza-ción, } \\
\text { la iniciativa } \\
\text { de futbol no } \\
\text { presenta un } \\
\text { vínculo claro }\end{array}$ & $\checkmark$ & $\mathrm{x}$ & $\begin{array}{l}\text { X Fomenta } \\
\text { deportes } \\
\text { emergentes } \\
\text { (existentes - } \\
\text { Parkour - Chase } \\
\text { Tag, Ultimate } \\
\text { Frisbee). } \\
\text { Plantea } \\
\text { modalidades } \\
\text { como Touch } \\
\text { Rugby y Rippa } \\
\text { Rugby }\end{array}$ & $\checkmark$ & $\mathrm{X}$ & $\mathrm{X}$ & $\mathrm{X}$ & $\begin{array}{l}\text { Sí, Amazon e Ebay (e- } \\
\text { commerce })\end{array}$ & $\begin{array}{l}\text { Haití } \\
\text { (aunque } \\
\text { domiciliado } \\
\text { en Florida) }\end{array}$ & $\begin{array}{l}\text { Nace a raíz del terremoto de } \\
\text { enero de } 2010 \text { en Haití. } \\
\text { Programa Haiti Soccer } 4 \\
\text { Development. Además se } \\
\text { habla de un programa: Haiti } \\
\text { Sports } 4 \text { Development }\end{array}$ \\
\hline $\begin{array}{l}\text { Rugby para } \\
\text { todos Chile }\end{array}$ & Sustantivo. & $\mathrm{X}$ & $\checkmark$ & $\checkmark$ & $\checkmark$ & $\checkmark$ & $\begin{array}{l}\text { X. } \\
\text { Se manifiesta } \\
\text { en contra de } \\
\text { los deportes } \\
\text { tradicionales, } \\
\text { sin } \\
\text { especificar a } \\
\text { qué se refiere }\end{array}$ & $\begin{array}{l}\checkmark \\
\text { Como } \\
\text { prestadores de } \\
\text { servicio social }\end{array}$ & $\begin{array}{l}\text { En busca de socios. Se } \\
\text { precisan montos, pero } \\
\text { no aparecen socios } \\
\text { activos }\end{array}$ & Chile & $\begin{array}{l}\text { Nace en 2015. Insatisfacción } \\
\text { con los deportes tradicionales } \\
\text { enseñados en las escuelas. Ha } \\
\text { logrado audiencia en la } \\
\text { Cámara de Diputados de } \\
\text { Chile. Metodología reflexiva, } \\
\text { incluyente y de no contacto, } \\
\text { RPT. }\end{array}$ \\
\hline
\end{tabular}




\section{Propuesta Tiro en Braille: una organización con metodología SDP para el cumplimiento de los ODS en México}

Ante la escasez de bibliografía SDP en general en América Latina y en específico en México, se decidió, como seguimiento al trabajo de Añorve (2014) sobre el deporte y los ODM, lanzar una iniciativa desde la Universidad de Guanajuato, durante el semestre agosto-diciembre de 2019.

Se tomaron como inspiración dos metodologías incluyentes: Ultimate Frisbee y el Fútbol Tres Tiempos (conocido en inglés como F3H). Para el caso del F3H se toman como referencia principal las adaptaciones que se hicieron a la metodología durante el Festival 2016 celebrado en Lyon (Francia), toda vez que, además de jugarse el torneo de forma mixta, se optó por no contar con equipos nacionales, por lo que el distintivo de los equipos era un color y no un Estado-nación (Segura e Islas, 2016).

La iniciativa, que a la postre dio origen a una organización-metodología, se basó en tres componentes:

1. La enseñanza-aprendizaje. Siendo el deporte un tema marginal dentro de las ciencias sociales, especialmente en México, el curso debía configurarse inicialmente como un Seminario Contemporáneo de Relaciones Internacionales. En la primera semana de agosto se dieron a conocer los contenidos, dejando claro que el curso ofrecía mucho más que solo deporte: contenía unidades sobre desarrollo, cooperación internacional, la Agenda 2030/ODS y la legislación mexicana. Por tratarse de una iniciativa institucional, lo que planteaba problemas de movilidad geográfica dentro y fuera de la ciudad, el curso se ofreció en línea.

2. La investigación. Estudiantes de licenciatura fueron invitados a publicar un capítulo en un libro colectivo (en proceso de edición), basándose en su experiencia de aprendizaje y deportiva durante el seminario en línea.

3. La experiencia deportiva práctica. Se lanzó una convocatoria para el diseño de una actividad deportiva. Se elaboró tomando en cuenta 12 de las 169 metas de los 17 ODS: 3.4, 4.5, 4.7, 5.1, 5.5, 5.c, 9.b, 10.2, 10.3, 11.4, 16.7 y 17.7. También se tomaron en cuenta la Constitución Política de los Estados Unidos Mexicanos (CPEUM) y la Ley General de Cultura Física y Deporte (LGCFD) vigentes. Un objetivo medular era alinear la relación entre el deporte y otros sectores, al tiempo que se dio importancia a aprendizajes y procesos SDP globales. De esta forma se logró un maridaje glocal entre el SDP y los ODS (dimensión internacional), y la CPEUM, la LGCFD, así como 
realidades de las universidades mexicanas y la necesidad de recuperar el patrimonio nacional.

La convocatoria, que sirvió para desarrollar y madurar una metodología, tuvo los siguientes requisitos: 1) al menos la mitad de los integrantes de un equipo debían ser mujeres; 2) al menos dos miembros de cada equipo debían participar dentro del seminario en línea; 3) cada equipo necesitaba un profesor-tutor; 4) la actividad deportiva diseñada debía ser apta para personas con discapacidades, 5) inspirarse en un juego/deporte precolombino o tradicional de México y 6) alinearse en la mayor medida posible con al menos tres legislaciones o agendas - el Plan Nacional de Desarrollo (PND) 2019-2024, la LGCFD, la CPEUM, la ley estatal vigente en materia deportiva o de educación física o la Agenda 2030 y los 17 ODS-; 7) los insumos para el juego y la señalización de la cancha de juego debían estar hechos con materiales reciclables y se prohibía explícitamente el uso de energías fósiles; 8) el diseño se alinearía con el mayor número posible de ODS; 9) la metodología sería progresista, incluyente y participativa —e.g., el Ultimate Frisbee (Griggs, 2011) o el F3H (Segura et al., 2018)—, y se aconsejaba la modalidad implementada durante el Festival de Lyon (Francia) en 2016; 10) se adjuntaría la carta de apoyo de al menos una pequeña o mediana empresa (pyme) domiciliada en la ciudad en la que se formulaba la propuesta; 11) habían de reflejarse los aprendizajes adquiridos dentro del seminario en línea.

El contenido tanto de la convocatoria como del proceso de selección de la propuesta ganadora tomó en cuenta, en todo momento, lo advertido por Schulenkorf et al. (2016), quienes apoyan la investigación colaborativa, que parte de la reflexividad del investigador y la deconstrucción de la retórica, el contexto y la narrativa del SDP (Darnell et al., 2018). Se tuvo en mente a Darnell, et al. (2018, p. 140), quienes advierten que hay temas subanalizados: discapacidad, igualdad de género y sustentabilidad. La inclusión e intersección deporte-género-discapacidades-deportes precolombinos/tradicionales es quizá el sello distintivo de Tiro en Braille, convertida en realidad en una metodología del mismo nombre, en homenaje al primer deporte de creación $100 \%$ estudiantil desarrollado a partir de la convocatoria de agosto de 2019.4

Las propuestas deportivas fueron evaluadas por un comité mixto (dos profesores, dos autoridades universitarias y dos alumnos). La ceremonia de premiación tuvo lugar el 25 de noviembre de 2019. Todos los equipos tendrían la posibilidad de publicar sus propuestas en un libro colectivo. Todos debían firmar una carta de intención en la que expresaban su disposición para jugar la propuesta ganadora durante el semestre enero- 
junio de 2020 (lo cual no ha sucedido por cauda del covid-19). Los participantes respondieron un cuestionario de 22 preguntas con el fin de monitorear el progreso/cambio en valores, actitudes y conductas observado a lo largo de esta experiencia SDP. Con el cuestionario se procura trascender las simples definiciones de «resultados» y la rendición de cuentas, para transitar de enfoques sumativos a enfoques formativos (Levermore, 2011).

Al seminario en línea se matricularon 33 estudiantes. El Campus Guanajuato se compone de seis divisiones y los estudiantes participantes pertenecían a dos de ellas: la División de Derecho, Política y Gobierno y la División de Ciencias Económicas y Administrativas. Por su parte, los 20 estudiantes que participaron en cinco equipos que diseñaron las propuestas que entraron al concurso pertenecían a cuatro divisiones (las dos mencionadas previamente, además de la División de Ingeniería y la División de Ciencias Naturales y Exactas).

Se recibieron cuatro propuestas y resultó ganadora Tiro en Braille. Además de cumplir con todos los lineamientos, esta tuvo la ventaja de ser diseñada explícitamente para la participación de personas con debilidad visual, con lo que se buscaba despertar conciencia de lo que esta discapacidad implica. Tiro en Braille requiere que todos los participantes usen un antifaz para «cegar» sus ojos. De forma simultánea juegan dos hombres y dos mujeres de cada equipo, mientras que un quinto jugador o jugadora auxilia verbalmente a sus compañeras(os) desde el perímetro de la cancha. Con el fin de orientar a los jugadores, la pelota (hecha con trapos y otros materiales reciclados) está rellena de cascabeles. Durante las sesiones de capacitación, el equipo ganador expresó que el diseño de Tiro en Braille está orientado a que se juegue en un entorno de relativo silencio, lo cual permite que los jugadores tengan una experiencia multisensorial.

Tiro en Braille se inspira en dos juegos precolombinos/tradicionales: el juego de pelota y el olote que vuela — desde luego, con adaptaciones significativas - . Debido a que se juega con los ojos vendados, la pelota rueda en lugar de volar, con lo que se busca evitar lesiones. Adicionalmente, por el hecho de jugar a ciegas, las diferencias físicas entre hombres y mujeres pueden verse reducidas. Finalmente, se destaca que no existen patrocinadores corporativos. Tiro en Braille logró cuatro apoyos, dos de pymes locales y dos de instituciones gubernamentales.

Al abordar los preparativos para la implementación, de forma colectiva recordamos las enseñanzas del F3H y del Ultimate Frisbee. Pese a contar con un reglamento detallado para Tiro en Braille, se acordó fijar un número limitado de reglas y vigilar su estricto cumplimiento; sin embargo, se permitirá que los equipos negocien reglas y acuerdos complementarios juego por juego, todo esto como parte del proceso de aprendizaje deliberativo. 
A 23 meses del lanzamiento de la convocatoria original que dio lugar a la creación de la actividad deportiva SDP Tiro en Braille, el status que guarda la iniciativa-organización-metodología rompe paradigmas. Aunque Tiro en Braille continúa sin poder jugarse debido a las restricciones de movilidad y presencialidad que covid-19 ha generado en México, la organización y la metodología no experimentan un impasse. La experiencia despertó el interés de la organización no gubernamental Fundación Vladimir Potanin en la Federación Rusa y ha sido compartida en sesiones virtuales en octubre de 2020 y abril de 2021. Más aún, la Benemérita y Centenaria Escuela Normal Oficial de Guanajuato (BCENOG), una institución de educación superior en la ciudad donde está domiciliada la organización-metodología, adaptó y emuló la convocatoria durante el semestre académico febrero-julio de 2021.

La participación de estudiantes de las licenciaturas en Inclusión Educativa, en Educación Primaria y en Educación Preescolar fue superior a la participación en la Universidad de Guanajuato en 2019. Se recibieron siete propuestas. Al igual que en la Universidad de Guanajuato, la actividad ganadora en la BCENOG será jugada en cuanto las condiciones sanitarias lo permitan. Así, lo que comenzó como una iniciativa para un semestre académico dentro de la Universidad de Guanajuato, para octubre de 2020 se convirtió en una organización-metodología incluida dentro de la plataforma Sportanddev.org.

\section{Discusión}

Sin duda, una de las ventajas que tuvo Tiro en Braille, por el momento de su nacimiento, es la visibilidad del movimiento SDP, la enorme difusión y resonancia que tienen los ODS y el creciente acervo de la bibliografía SDP publicada en otras latitudes; especialmente, se benefició de la bibliografía crítica, que trata al SDP dentro de la matriz de poder-privilegio-dominio (Hartmann y Kwauk, 2011) y aborda la forma en que el deporte reproduce desigualdades, injusticias y marginalidad (Kincheloe, 2008), pero también considera que el deporte ofrece un núcleo fundamental para repensar y repracticar las políticas del desarrollo, apoyando procesos decolonizadores y libertarios (Darnell y Hayhurst, 2011).

$\mathrm{Al}$ analizar el contenido de las tablas 1-3, surgen diversos hallazgos. Primero, en la región de América Latina y el Caribe hay una cantidad importante de países o territorios sin organizaciones SDP registradas ante Sportanddev.org: Guatemala, Belice, Cuba, Antigua y Barbuda, Aruba, Bahamas, Granada, Guadalupe, Islas Caimán, Islas Turcas y Caicos, Islas Vírgenes, Martinica, República Dominicana, San Bartolomé, San Cristóbal y Nieves, San Vicente y las Granadinas y Guyana Francesa. Claramente, el movimiento SDP aún tiene territorios hacia los cuales expandirse. 
Segundo, excluyendo Tiro en Braille, existen nueve organizaciones en América Latina y el Caribe, y una en México, que recuperan la Agenda 2030/ODS; sin embargo, solo seis en la región y una en México lo hacen de forma sustantiva; es decir, solo el 7,69\% de las organizaciones regionales otorgan centralidad a esta agenda global, lo cual está en línea con la casi nula atención otorgada al SDP en la región que se expuso en el primer apartado.

Tercero, la riqueza de Tiro en Braille no deriva en realidad del componente deportivo (no se ha jugado debido al covid-19). En realidad, su principal riqueza es la metodología desarrollada. Puede verse en las tablas 2 y 3 que solo tres organizaciones (además de Tiro en Braille) cuentan con una metodología - Colombianitos (Juguemos por la Paz), Futbol con Corazón (Futbol por la Paz) y Rugby para Todos Chile (RPT) - y ninguna de las tres propone la creación de una actividad deportiva (dos de ellas parten de una forma distinta de implementar el futbol). Ciertamente, destacan la adaptación para personas discapacitadas en Rugby para Todos y la inclusión y las metodologías reflexivas en Futbol con Corazón, cuya metodología en gran parte guarda paralelismos con el F3H (Segura e Islas, 2016; Segura et al., 2018). Rugby para Todos Chile intenta disminuir/cancelar el contacto físico y con ello el riesgo de lesiones en un deporte como el rugby, algo similar a lo planteado en el Ultimate Frisbee (Griggs, 2011). No obstante sus virtudes, ninguna de estas tres organizaciones-metodologías plantea los tres componentes de OM —-docencia, investigación y creación de una actividad deportiva SDP alineada específicamente con los ODS- La atención paralela de población universitaria (ODS 4), mujeres (ODS 5), discapacitados (ODS 10) y la búsqueda de asociaciones con pymes locales (ODS 17) otorgan a Tiro en Braille un carácter holístico.

Cuarto, dentro de Sportland (Giulianotti et al., 2019), resulta claro que son los países más pobres y los segmentos poblaciones más marginados los destinatarios de la mayoría de las iniciativas SDP. Debido a que se ha abandonado el enfoque basado en derechos humanos (Canan y Starepravo, 2019), poblaciones relativamente privilegiadas como las universitarias han sido dejadas de lado. Aunque las tablas 2 y 3 identifican la participación universitaria, esta se contempla como potencial para el caso de la Caribbean Sport and Development Agency (CSDA), está limitada a prestadores de servicio social (Rugby para Todos Chile) o bien plantea su participación dentro de una organización ya constituida con una práctica deportiva ya diseñada (es el caso de Capoeira Congo y Capoeira para Todos, en México). En ningún caso los estudiantes son el centro de la organización, como sí sucede con Tiro en Braille.

Finalmente, respecto a la meta 4.7 del ODS 4, que hace referencia a la valoración de la diversidad cultural y la contribución de la cultura al desarrollo sostenible, además de Tiro en Braille, solo las dos organizaciones que trabajan con capoeira —una en México y la otra en Brasil (y África)—, además de Promotora Mexicana de Valores Deportivos A. C., trabajan en la recuperación de deportes tradicionales. En el caso de la última, no 
queda claro de qué forma se operan los programas SDP; da más bien la impresión de tratarse de un portal para la venta de artículos deportivos disfrazado de organización SDP.

Tiro en Braille fue concebida dentro de estudios SDP que han contrastado los esfuerzos y aproximaciones desde arriba (top-down) con los enfoques de iniciativas desde abajo (grassroots) (Norwegian Ministry of Foreign Affairs, 2006). Este paradigma para el desarrollo ha puesto un creciente énfasis en las relaciones sociales y las redes de desarrollo comunitario de abajo arriba (bottom-up community development), más que en iniciativas top-down (Coalter, 2010).

Tiro en Braille impulsa y difunde este paradigma; fue concebido después de leer un texto medular de Hatton (2015) que señala la importancia del monitoreo y la evaluación. A través de su segundo componente —la investigación-, Tiro en Braille se compromete a demostrar lo que sugiere Hatton (2015, p. 16): su relevancia más allá del contexto inicial (geográfico), su simplicidad relativa (a la que agrega su bajo costo), su superioridad frente a otras alternativas (al menos en el diseño, Tiro en Braille es una iniciativa de frontera).

\section{Conclusiones}

Si bien es cierto que el SDP y, en menor grado, su vinculación con los ODS ya aparece en la literatura académica dentro del Norte global, en América Latina y en México fluctúan entre la inexistencia y la escasez. Dentro del Norte global, la implementación de proyectos SDP no está libre de críticas. El futbol, pese a no ser monopólico, sigue dominando Sportland. Aunque la participación de organizaciones SDP en América Latina y el Caribe es nimia, a diferencia de la atención académica, existen 79 organizaciones domiciliadas en la región dentro de la plataforma global líder SDP Sportanddev.org, siete de las cuales se encuentran en México. De estas, menos del 10\% presenta un vínculo con la Agenda 2030/ODS; más aún, seis de las diez organizaciones que lo hacen se enfocan en el futbol.

A pesar de que tres organizaciones regionales han desarrollado una metodología alineada con algunos de los ODS, ninguna presenta un diseño holístico de inclusiones múltiples: algunas suelen centrarse en el género, otras en la discapacidad, las menos (tres) plantean la recuperación de deportes tradicionales y la participación de estudiantes universitarios. Seis de las diez organizaciones regionales confieren un papel central a los patrocinadores corporativos, con lo cual corren el riesgo de inviabilidad en caso de que los intereses de las organizaciones entren en conflicto con los intereses corporativos. Este dominio corporativo suele enfocarse en aproximaciones de tipo sport plus, con lo cual los proyectos top-bottom suelen dominar. 
Pese a la escasa atención académica que se da al SDP en América Latina y el Caribe, hay publicaciones (Levoratti y Zambaglione, 2016) y proyectos gubernamentales en materia de deporte social (Presidencia de la República, 2019) que posibilitan, con la debida presentación de ejemplos, emprender organizaciones SDP vinculadas a los ODS en la región, partiendo de lo que se ha logrado avanzar en materia de deporte social.

Tiro en Braille representa una metodología, más que una actividad deportiva, que ha tenido la fortuna de nutrirse de la bibliografía existente para poder plantear alternativas SDP bajo un enfoque plus sport. Entre las ventajas que presenta, destaca su diseño, deliberado, para escapar a presiones corporativas, la posibilidad de ser replicado en prácticamente cualquier entorno universitario, su bajo costo, el empoderamiento real de los participantes (estudiantes universitarios) desde la concepción de la actividad, además de evidenciar su actuación a través de la plataforma global Sportanddev.org.

\section{Referencias}

Asociación Latinoamericana de Estudios Socioculturales del Deporte. (2010). História ALESDE. Recuperado de http://www.alesde.ufpr.br/historia.html.

Añorve, D. (2014). La actividad física y el deporte en la edificación de una ciudadanía democrática y en los Objetivos de Desarrollo del Milenio: el caso de Guanajuato. En J. Aguilar (coord.), Libertades y participación política: Desafíos para la consolidación democrática en Guanajuato (pp. 355-409). México: Fontamara.

Añorve, D. (2021). Sport for development and peace (SDP): A proposal for meaningful inclusion leading to sustained change. En P. Liamputtong (ed.), Handbook of social inclusion (pp. 1-18). Switzerland: Springer Nature.

Barboza, S., y Marinho, F. (2019). Projetos e programas sociais esportivos no Brasil: Antecedentes históricos e reflexividades social. Journal of the Latin American Sociocultural Studies of Sport, 10(1), 33-52.

Burnett, C. (2015). Assessing the sociology of sport: On sport for development and peace. International Review for the Sociology of Sport, 50(4-5), 385-390.

Canan, F., y Starepravo, F. (2019). 0 significado e a abrangência do direito ao esporte a partir de documentos internacionais. Fair Play: Revista de Filosofía, Ética y Derecho del Deporte, 15, 1-36.

Coalter, F. (2007). Sport a wider social role: Whose keeping the score? Londres: Routledge. 
Coalter, F. (2010). The politics of sport-for-development: Limited focus programmes and broad gauge problems? International Review for the Sociology of Sport, 45(3), 295-314.

Darnell, S., Chawansky, M., Marchesseault, D., Holmes, M., y Hayhurst, L. (2018). The state of play: Critical sociological insights into recent "Sport for Development and Peace" research. International Review for the Sociology of Sport, 53(2), 133 151.

Darnell, S., y Hayhurst, L. (2011). Sport for decolonization: Exploring a new praxis of sport for development. Progress in Development Studies, 11(3): 183-96.

Giulianotti, R., Coalter, F., Collison, H., y Darnell, S. (2019). Rethinking sportland: A new research agenda for the sport for development and peace sector. Journal of Sport and Social Issues, 6, 411-437.

Griggs, G. (2011). This must be the only sport in the world where most of the players don't know the rules: Operationalizing selfrefereeing and the spirit of the game in UK Ultimate Frisbee. Sport in Society: Cultures, Commerce, Media, Politics, 14(1), 97-110.

Guttmann, A. (1978). From ritual to record: The nature of modern sports. Nueva York: Columbia University Press.

Hartmann, D., y Kwauk, C. (2011). Sport and development: An overview, critique, and reconstruction. Journal of Sport and Social Issues, 35(3), 284-305.

Hatton, D. (2015). Shaping the «Sport for Development» agenda post-2015. Londres: Infocus. Recuperado de https://www.sport-for-development.com/imglib/downloads/infocus2015-shaping-the-s4d-agenda-post-2015.pdf.

Inglés, E., y Puig, N. (2013). Estrategias de gestión de la práctica deportiva en el medio natural: Una propuesta de investigación. Journal of the Latin American Socio-Cultural Studies of Sport, 3(1), 4-19.

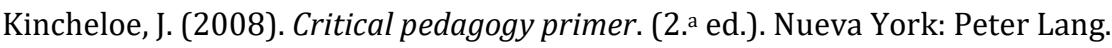

Kravchychyn, C., De Souza, J., Starepravo, F., Parra, I., y Bassóli, A. (2019). Projetos e programas sociais esportivos no Brasil: Antecedentes históricos e reflexividade social. Journal of the Latin American Socio-cultural Studies of Sport, 10(1), 53-68.

Levermore, R. (2011). Evaluating sport-for-development: Approaches and critical issues. Progress in Development Studies, 11(4): 339-353.

Levoratti, A., y Zambaglione, D. (2016). Reflexiones sobre el deporte para la inclusión a partir de los debates para modificar la Ley 20655, «Promoción de las actividades deportivas en todo el país», y su inscripción universitaria en la formación de profesores de Educación Física. En C. Guzmán (comp.), Deporte, inclusión social y experiencias comunitarias en América Latina (pp. 107-112). Villavicencio: Unillanos. 
Lindsey, I., y Darby, P. (2019). Sport and the sustainable development goals: Where is the policy coherence? International Review for the Sociology of Sport, 54(7), 793812.

Marchi, W. (2015). Assessing the sociology of sport: On Brazil and Latin American perspectives. International Review for the Sociology of Sport, 50(4-5), 530-535.

Melo, M. (2018). Ano internacional do esporte e educação física: 0 sistema ONU e a pedagogia da hegemonia. Educación Física y Ciencia, 20, 1-10.

Metropole Lémanique. (2017). Achieving SDGs through sport: Partnerships and institutional responses for greater coherence and effectiveness. 5 de octubre, Lausanne. Recuperado de http://www.metropole-lemanique.ch/docs/05-10-2017-final-report.pdf.

Mwaanga, O., y Mwansa, K. (2014). Indigenous discourses in sport for development and peace: A case study of the Ubuntu cultural philosophy in EduSport Foundation, Zambia. En N. Schulenkorf y D. Adair (eds.), Global sport-for-development: Critical perspectives (pp. 115-133). Nueva York: Palgrave MacMillan.

Norwegian Ministry of Foreign Affairs. (2006). Strategy for Norway's culture and sports co-operation with Countries in the South. Oslo: Norwegian Ministry of Foreign Affairs.

Nunes, A., Lee, K., y O’Riordan, T. (2016). The importance of an integrating framework for achieving the sustainable development goals: The example of health and wellbeing. BMJ Global Health, 1, 1-12.

Paramio-Salcines, J., Babiak, K., y Walters, G. (2013). Routledge handbook of sport and corporate social responsibility. Londres: Routledge.

Presidencia de la República. (2019). Plan Nacional de Desarrollo (2019-2024). Ciudad de México: Gobierno de México.

Schulenkorf, N., Sherry, E., y Rowe, K. (2016). Sport for development: An integrated literature review. Journal of Sport Management, 30, 22-39.

Segura, F., e Islas, A. (2016). El fútbol en tres tiempos sociales. Podium, Sport, Leisure \& Tourism Review, 5(2), 1-14.

Segura, F., Norman, M., y Jaccoud, C. (2018). Encounters on the field: Observations of the football-3-halves festival during the Euro Cup 2016. Sociology of Sport Journal, 35(4), 367-374.

Sundelin, R., Gilmartin, M., y Forman, A. (2017, 8 de junio). Sport 4 The global goals pledge. The Global Goals. Recuperado de https://www.sportanddev.org/sites/default/files/files/sport4sdgs_oslo_june2017.pdf.

Toledo, R. (2019). Esporte, direitos sociais e políticas públicas no Brasil: Desenho institucional em perspectiva comparada. Journal of the Latin American Socio-cultural Studies of Sport, 10(1), 20-32. 
United Nations. (2003). Sport for development and peace: towards achieving the millennium development goals. Report from the United Nations inter-agency task force on sport for development and peace. Ginebra: United Nations.

United Nations General Assembly. (2015). Transforming our world: The 2030 agenda for sustainable development. Recuperado de http://www.un.org/ga/search/ view_doc.asp?symbol=A/RES/70/1\&Lang=E. 